\title{
A higher-order accurate operator splitting spectral method for the Wigner-Poisson system
}

\author{
Zhenzhu Chen ${ }^{\dagger, \ddagger} \quad$ Sihong Shao ${ }^{\ddagger}, *$
}

May 31, 2021

\begin{abstract}
An accurate description of 2-D quantum transport in a double-gate metal oxide semiconductor filed effect transistor (dgMOSFET) requires a high-resolution solver to a coupled system of the 4-D Wigner equation and 2-D Poisson equation. In this paper, we propose an operator splitting spectral method to evolve such Wigner-Poisson (WP) system in 4-D phase space with high accuracy. After an operator splitting of the Wigner equation, the resulting two sub-equations can be solved analytically with spectral approximation in phase space. Meanwhile, we adopt a Chebyshev spectral method to solve the Poisson equation. Spectral convergence in phase space and a fourth-order accuracy in time are both numerically verified. Finally, we apply the proposed solver into simulating dgMOSFET, develop the steady states from long-time simulations and obtain numerically converged current-voltage (I-V) curves.
\end{abstract}

Keywords: Wigner-Poisson system; operator splitting; spectral method; MOSFET; I-V curve; RTD

\section{Introduction}

In the last two decades, the Wigner function approach $[1,2]$ has provided a powerful tool for studying quantum effect in various electronic devices, such as the resonant tunneling diodes (RTDs) [3] and the metal oxide semiconductor filed effect transistors (MOSFETs) [4]. A coupled system of the Wigner equation and the Poisson equation is usually adopted for taking the space charge effects into account. Finite difference methods were often used to obtain numerical solutions of the Wigner equation $[5,6]$ as well as of the Wigner-Poisson (WP) system [7, 8], and several spectral methods were also tried $[3,9,10]$. In order to accurately capture $2-\mathrm{D}$ quantum transport in a double-gate MOSFET (dgMOSFET), the WP system in 4-D phase space is required to be integrated with high resolution. However, all above-mentioned numerical methods

\footnotetext{
$\dagger$ Institute of Applied Physics and Computational Mathematics, Beijing 100094, China .

${ }^{\ddagger}$ LMAM and School of Mathematical Sciences, Peking University, Beijing 100871, China.

*To whom correspondence should be addressed. Email: sihong@math.pku.edu.cn
} 
were implemented in 2-D phase space, and highly accurate deterministic numerical methods for the WP system in 4-D phase space are very few up to now. This paper is intended to fill this gap by exploiting a recently developed operator splitting spectral method for the 4-D Wigner equation in quantum double-slit interference [11]. Specifically, we will take advantage of the operator splitting spectral method to solve the 4-D Wigner equation, in which the semi-discrete models resulted from spectral expansion in phase space for the sub-equations have analytical solutions, and continue to use a Chebyshev spectral method to solve the 2-D Poisson equation.

Detailed benchmark tests are performed with the Gaussian barrier scattering in 2$\mathrm{D}$ and 4-D phase space, and demonstrate that the proposed operator splitting spectral method indeed has a spectral accuracy in phase space and a fourth-order accuracy in time. We also show that the electric field induced by the space charge has a great effect on the rate of quantum tunneling. After calibration, we apply our high-resolution solver into simulating RTD and dgMOSFET. Numerical experiments show that the steady states can be well developed from long-time simulations and the corresponding current-voltage $(\mathrm{I}-\mathrm{V})$ curves are numerically converged as the number of collocation points increases.

The remainder of this paper is organized as follows. Section 2 briefs the WP system. Section 3 presents the operator splitting spectral method. Section 4 conducts benchmark tests with the Gaussian barrier scattering. Simulations and discussions of RTD and dgMOSFET are given in Sections 5 and 6, respectively. The paper is concluded in Section 7 with a few remarks.

\section{The Wigner-Poisson system}

The Wigner function $f(\boldsymbol{x}, \boldsymbol{k}, t)$ living in $2 d$-D phase space: $(\boldsymbol{x}, \boldsymbol{k}) \in \mathbb{R}^{2 d}$ with position $\boldsymbol{x} \in \mathbb{R}^{d}$ and wavevector $\boldsymbol{k} \in \mathbb{R}^{d}$, obeys the following Wigner equation [1]

$$
\frac{\partial}{\partial t} f(\boldsymbol{x}, \boldsymbol{k}, t)+\frac{\hbar \boldsymbol{k}}{m} \cdot \nabla_{\boldsymbol{x}} f(\boldsymbol{x}, \boldsymbol{k}, t)=\Theta_{V}[f](\boldsymbol{x}, \boldsymbol{k}, t),
$$

where $d$ gives the dimension of position space, $t$ denotes the time, $\hbar$ is the reduced Planck constant, $m$ is the mass, and $\Theta_{V}[f]$ is the so-called nonlocal pseudo-differential operator containing all the quantum information:

$$
\begin{aligned}
\Theta_{V}[f](\boldsymbol{x}, \boldsymbol{k}, t) & =\int_{\mathbb{R}^{d}} \mathrm{~d} \boldsymbol{k}^{\prime} f\left(\boldsymbol{x}, \boldsymbol{k}^{\prime}, t\right) V_{w}\left(\boldsymbol{x}, \boldsymbol{k}-\boldsymbol{k}^{\prime}, t\right), \\
V_{w}(\boldsymbol{x}, \boldsymbol{k}, t) & =\frac{1}{\mathrm{i} \hbar(2 \pi)^{d}} \int_{\mathbb{R}^{d}} \mathrm{~d} \boldsymbol{y} \mathbb{e}^{-\dot{\mathrm{i}} \boldsymbol{k} \boldsymbol{y}}\left[V\left(\boldsymbol{x}+\frac{\boldsymbol{y}}{2}, t\right)-V\left(\boldsymbol{x}-\frac{\boldsymbol{y}}{2}, t\right)\right] .
\end{aligned}
$$

Here $V(\boldsymbol{x}, t)$ gives the external potential, and can be rewritten into $V(\boldsymbol{x}, t)=V_{b}(\boldsymbol{x})+$ $V_{e}(\boldsymbol{x}, t)$ when taking the space charge effects into account, where $V_{b}(\boldsymbol{x})$ denotes the conduction band potential and $V_{e}(\boldsymbol{x}, t)$ the effective electric potential. Actually, $V_{e}(\boldsymbol{x}, t)$ can be determined by a Poisson equation with the electron density as its source term:

$$
-\nabla_{\boldsymbol{x}}\left(\epsilon(\boldsymbol{x}) \nabla_{\boldsymbol{x}}\right) V_{e}(\boldsymbol{x}, t)=-n(\boldsymbol{x}, t)+N_{d}(\boldsymbol{x})
$$


where $\epsilon(\boldsymbol{x})$ is the dielectric constant, $N_{d}(\boldsymbol{x})$ is the doping density and $n(\boldsymbol{x}, t)$ denotes the density of electrons given by

$$
n(\boldsymbol{x}, t)=\int_{\mathbb{R}^{d}} \mathrm{~d} \boldsymbol{k} f(\boldsymbol{x}, \boldsymbol{k}, t) .
$$

And, the current density $J(\boldsymbol{x}, t)$ can be further calculated by

$$
J(\boldsymbol{x}, t)=\int_{\mathbb{R}^{d}} \mathrm{~d} \boldsymbol{k} \frac{\hbar \boldsymbol{k}}{m} f(\boldsymbol{x}, \boldsymbol{k}, t) .
$$

In this work, we focus on developing a high-resolution solver for the WP system in 4 -D phase space (i.e., $\boldsymbol{d}=2$ ) and let $\boldsymbol{x}=(x, z), \boldsymbol{k}=\left(k_{x}, k_{z}\right)$. In particular, our target is to simulate the dgMOSFET and the working-equations read

(a) $\frac{\partial}{\partial t} f(\boldsymbol{x}, \boldsymbol{k}, t)+\frac{\hbar \boldsymbol{k}}{m} \cdot \nabla_{\boldsymbol{x}} f(\boldsymbol{x}, \boldsymbol{k}, t)=\Theta_{V}[f](\boldsymbol{x}, \boldsymbol{k}, t)$,

(b) $f(\boldsymbol{x}, \boldsymbol{k}, t=0)=f_{0}(\boldsymbol{x}, \boldsymbol{k})$,

(c) $f\left(x_{l}, z, \boldsymbol{k}, t\right)=f_{l x}(z, \boldsymbol{k}, t), \quad k_{x}>0, \quad f\left(x_{r}, z, \boldsymbol{k}, t\right)=f_{r x}(z, \boldsymbol{k}, t), \quad k_{x}<0$,

(d) $f\left(x, z_{l}, \boldsymbol{k}, t\right)=f_{l z}(x, \boldsymbol{k}, t), \quad k_{z}>0, \quad f\left(x, z_{r}, \boldsymbol{k}, t\right)=f_{r z}(x, \boldsymbol{k}, t), \quad k_{z}<0$,

(e) $-\Delta V_{e}(x, z, t)=\left(-n(x, z, t)+N_{D}^{+}-N_{A}^{-}\right) / \epsilon$,

(f) $V_{e}\left(x, z_{l}, t\right)=0, \quad V_{e}\left(x, z_{r}, t\right)=-V_{g}$,

(g) $\frac{\partial V_{e}(x, z, t)}{\partial x}=0$ at $x_{l}, x_{r}$

where we have chosen the commonly used inflow boundary conditions for the Wigner equation [5, 12], and mixed boundary conditions for the Poisson equation: the Dirichlet boundary in $z$-direction plus the Neumann boundary in $x$-direction, the computational domain for position is $\Omega_{\boldsymbol{x}}=\left[x_{l}, x_{r}\right] \times\left[z_{l}, z_{r}\right], V_{g}$ gives the gate voltage, and $N_{D}^{+}$and $N_{A}^{-}$are the ionized donor and acceptor impurities doping concentrations, respectively.

\section{$3 \quad$ Numerical methods}

Considering the decay property of the Wigner function when $|\boldsymbol{k}| \rightarrow+\infty$, a simple nullification outside a sufficiently large $\boldsymbol{k}$-domain $\Omega_{\boldsymbol{k}}$ is usually adopted $[11,13,14]$, thus we are in fact using a truncated pseudo-differential operator $\Theta_{V}^{T}[f]$ in $\boldsymbol{k}$-space as follows

$$
\begin{aligned}
\Theta_{V}^{T}[f]\left(x, z, k_{x}, k_{z}, t\right) & =\iint_{\Omega_{k}} \mathrm{~d} k_{x}^{\prime} \mathrm{d} k_{z}^{\prime} f\left(x, z, k_{x}^{\prime}, k_{z}^{\prime}, t\right) \tilde{V}_{w}\left(x, z, k_{x}-k_{x}^{\prime}, k_{z}-k_{z}^{\prime}, t\right), \\
\tilde{V}_{w}\left(x, z, k_{x}, k_{z}, t\right) & =\frac{\Delta y_{x} \Delta y_{z}}{\dot{\mathrm{i}} \hbar(2 \pi)^{2}} \sum_{\mu=-\infty}^{+\infty} \sum_{\nu=-\infty}^{+\infty} D_{V}\left(x, z, y_{x, \mu}, y_{z, \nu}, t\right) \mathrm{e}^{-\dot{\mathrm{i}} k_{x} y_{x, \mu}-\dot{\mathrm{i}} k_{z} y_{z, \nu}}, \\
D_{V}\left(x, z, y_{x}, y_{z}, t\right) & =V\left(x+\frac{y_{x}}{2}, z+\frac{y_{z}}{2}, t\right)-V\left(x-\frac{y_{x}}{2}, z-\frac{y_{z}}{2}, t\right),
\end{aligned}
$$

where $\Omega_{\boldsymbol{k}}=\left[k_{x, \min }, k_{x, \max }\right] \times\left[k_{z, \min }, k_{z, \max }\right]$ and $y_{x, \mu}=\mu \Delta y_{x}, y_{z, \nu}=\nu \Delta y_{z}$ with $\Delta y_{x}$, $\Delta y_{z}$ being the spacing, which satisfy $\Delta y_{i}=2 \pi / L_{k_{i}}$ with $L_{k_{i}}=k_{i, \max }-k_{i, \min }$ for $i=x, z$ in this paper. 


\subsection{Solving the 4-D Wigner equation}

The operator splitting spectral method developed in [11] for simulating the quantum double-slit interference is employed here for solving the 4-D Wigner equation. A brief description is given below and the interested readers are referred to [11] for more details.

An $s$-stage exponential operator splitting method for the Wigner equation Eq. (1) reads

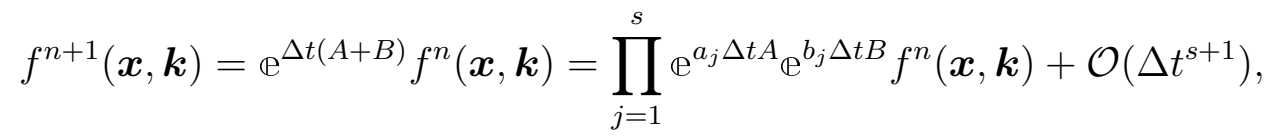

where $f^{n}(\boldsymbol{x}, \boldsymbol{k}):=f\left(\boldsymbol{x}, \boldsymbol{k}, t^{n}\right)$ denotes the exact solution at time $t^{n}:=n \Delta t$ and

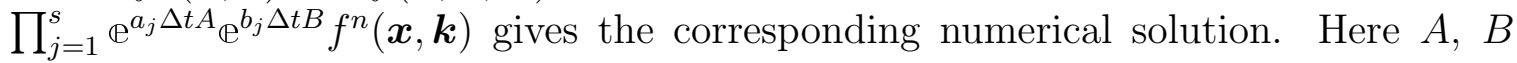
are the convection operator and pseudo-differential operator, which correspond to two sub-equations of the Wigner equation, respectively:

$$
\left\{\begin{array}{l}
(\mathrm{A}) \frac{\partial}{\partial t} f(\boldsymbol{x}, \boldsymbol{k}, t)=-\frac{\hbar \boldsymbol{k}}{m} \cdot \nabla_{\boldsymbol{x}} f(\boldsymbol{x}, \boldsymbol{k}, t), \\
\text { (B) } \frac{\partial}{\partial t} f(\boldsymbol{x}, \boldsymbol{k}, t)=\Theta_{V}^{T}[f](\boldsymbol{x}, \boldsymbol{k}, t)
\end{array}\right.
$$

We adopt the advective approach to march the sub-equation (A) in Eq. (10) strictly along the characteristic lines as follows

$$
f^{n+1}(\boldsymbol{x}, \boldsymbol{k})=\mathbb{e}^{\Delta t A} f^{n}(\boldsymbol{x}, \boldsymbol{k})=f^{n}(\boldsymbol{x}-\boldsymbol{v} \Delta t, \boldsymbol{k}), \quad \boldsymbol{v}=\frac{\hbar \boldsymbol{k}}{m},
$$

and the Chebyshev expansion of the Wigner function with respect to $\boldsymbol{x}$ is used to obtain function values at shifted points.

Motivated by the intrinsic nature of Fourier transformation contained in the pseudodifferential term Eq. (8), we use a Fourier spectral method to solve the sub-equation (B) in Eq. (10). The interpolation operator $\mathcal{I}_{\boldsymbol{k}, \boldsymbol{N}}$ reads

$$
\mathcal{I}_{\boldsymbol{k}, N} f(\boldsymbol{x}, \boldsymbol{k}, t)=\sum_{\nu_{x}=-N_{x} / 2+1}^{N_{x} / 2+1} \sum_{\nu_{z}=-N_{z} / 2+1}^{N_{z} / 2} a_{\nu_{x}, \nu_{z}}(\boldsymbol{x}, t) \psi_{\nu_{x}}\left(k_{x}\right) \psi_{\nu_{z}}\left(k_{z}\right)
$$

where $\psi_{\nu_{i}}\left(k_{i}\right)=\mathbb{e}^{2 \pi \mathrm{i} \nu_{i}\left(k_{i}-k_{i, \min }\right) / L_{k_{i}}}$ are the Fourier basis functions and $N_{i}$ the number of collocation points in $k_{i}$-space for $i=x, z$. Substituting the interpolation function $\mathcal{I}_{\boldsymbol{k}, \boldsymbol{N}} f(\boldsymbol{x}, \boldsymbol{k}, t)$ into the pseudo-differential term Eq. (8) also yields spectral approximation

$$
\begin{aligned}
\Theta_{V}^{T}[f](\boldsymbol{x}, \boldsymbol{k}, t) & \approx \sum_{\nu_{x}=-N_{x} / 2+1}^{N_{x} / 2+1} \sum_{\nu_{z}=-N_{z} / 2+1}^{N_{z} / 2} c_{\nu_{x}, \nu_{z}}(\boldsymbol{x}) a_{\nu_{x}, \nu_{z}}(\boldsymbol{x}, t) \psi_{\nu_{x}}\left(k_{x}\right) \psi_{\nu_{z}}\left(k_{z}\right), \\
c_{\nu_{x}, \nu_{z}}(x, z) & =\frac{1}{\hbar} D_{V}\left(x, z, y_{x, \nu_{x}}, y_{z, \nu_{z}}\right) .
\end{aligned}
$$


Accordingly, the orthogonal relation of the Fourier basis functions implies

$$
\frac{\partial}{\partial t} a_{\nu_{x}, \nu_{z}}(\boldsymbol{x}, t)=c_{\nu_{x}, \nu_{z}}(\boldsymbol{x}) a_{\nu_{x}, \nu_{z}}(\boldsymbol{x}, t)
$$

the solution of which has the following explicit form

$$
a_{\nu_{x}, \nu_{z}}^{n+1}(\boldsymbol{x})=\mathbb{e}^{c_{\nu_{x}, \nu_{z}}(\boldsymbol{x}) \Delta t} a_{\nu_{x}, \nu_{z}}^{n}(\boldsymbol{x}) .
$$

To match with the spectral accuracy in phase space, we adopt a fourth-order splitting scheme with $s=4$ in Eq. (9):

$$
\begin{aligned}
& a_{1}=a_{4}=\frac{1}{2(2-\sqrt[3]{2})}, \quad a_{2}=a_{3}=\frac{1-\sqrt[3]{2}}{2(2-\sqrt[3]{2})}, \\
& b_{1}=b_{3}=\frac{1}{2-\sqrt[3]{2}}, \quad b_{2}=-\frac{\sqrt[3]{2}}{2-\sqrt[3]{2}}, \quad b_{4}=0 .
\end{aligned}
$$

In subsequent numerical experiments, we usually choose $N_{x}=N_{z}:=N$ for convenience.

\subsection{Solving the 2-D Poisson equation}

The Chebyshev expansion in $\boldsymbol{x}$ direction continues to be used to solve the Poisson equation in $\left[x_{l}, x_{r}\right] \times\left[z_{l}, z_{r}\right]$ :

$$
\Delta V(x, z)=r(x, z)
$$

with mixed boundary conditions:

$$
V\left(x, z_{l}\right)=0, \quad V\left(x, z_{r}\right)=-V_{g}, \quad \frac{\partial V\left(x_{l / r}, z\right)}{\partial x}=0 .
$$

Then, $V(x, z), r(x, z), \Delta V(x, z)$ and $\partial_{x} V(x, z)$ are approximated by the truncated Chebyshev series as follows

$$
\begin{aligned}
V(x, z) & \approx \sum_{n=0}^{M} \sum_{m=0}^{M} a_{n m} \phi_{n}(x) \phi_{m}(z), \quad r(x, z) \approx \sum_{n=0}^{M} \sum_{m=0}^{M} b_{n m} \phi_{n}(x) \phi_{m}(z), \\
\Delta V(x, z) & \approx \sum_{n=0}^{M} \sum_{m=0}^{M} a_{n m}^{(2)} \phi_{n}(x) \phi_{m}(z), \quad \partial_{x} V(x, z) \approx \sum_{n=0}^{M} \sum_{m=0}^{M} a_{n m}^{(1)} \phi_{n}(x) \phi_{m}(z),
\end{aligned}
$$

where $\phi_{n}(x)$ and $\phi_{m}(z)$ are the Chebyshev polynomials of the first kind.

For simplicity, we suppose that the numbers of collocation points in $x$ and $z$ directions are even and the same, denotes by $M$. It can be readily verified that the expansion coefficients satisfy the following relationships

$$
\begin{aligned}
& a_{n m}^{(2)}=\frac{1}{c_{n}} \sum_{p=n+2: 2: M} p\left(p^{2}-n^{2}\right) a_{p m}+\frac{1}{c_{m}} \sum_{q=m+2: 2: M} q\left(q^{2}-m^{2}\right) a_{n q}, \\
& a_{n m}^{(1)}=\frac{1}{c_{n}} \sum_{p=n+1: 2: M} 2 p a_{p m} .
\end{aligned}
$$


The collocation equations for $\left\{a_{n m}\right\}$ that follow from Eqs. (13)-(14) are then

$$
\begin{gathered}
\frac{1}{c_{n}} \sum_{p=n+2: 2: M} p\left(p^{2}-n^{2}\right) a_{p m}+\frac{1}{c_{m}} \sum_{q=m+2: 2: M} q\left(q^{2}-m^{2}\right) a_{n q}=b_{n m}, \quad 0 \leq n, m \leq M-2, \\
\sum_{n \text { odd }} n^{2} a_{n m}=0, \quad \sum_{n \text { even }} n^{2} a_{n m}=0, \quad m=0,1, \ldots, M, \\
\sum_{m \text { odd }} a_{n m}=b_{n} / 2, \quad \sum_{m \text { even }} a_{n m}=b_{n} / 2, \quad n=0,1, \ldots, M,
\end{gathered}
$$

where $b_{0}=-V_{g}$ and $b_{i}=0$ for $i \neq 0$

We define the column vectors $X_{i}, B_{i}$ for $i=0,1, \ldots, M$ by

$$
\begin{aligned}
X_{i} & =\left(a_{0 i}, a_{1 i}, \cdots, a_{M-1, i}, a_{M i}\right)^{\top}, \quad i=0,1, \ldots, M, \\
B_{0} & =\left(-V_{g} / 2,0, \cdots, 0,0\right)^{\top}, \quad B_{1}=\left(-V_{g} / 2,0, \ldots, 0,0\right)^{\top}, \\
B_{i} & =\left(0,0, b_{0 i}, b_{1 i}, \cdots, b_{M-3, i}, b_{M-2, i}\right)^{\top}, \quad i=2,3, \ldots, M,
\end{aligned}
$$

and let $P$ be the $(M+1) \times(M+1)$ matrix as shown in Eq. (31) in Appendix. Consequently, Eqs. (15)-(17) can be rewritten into

$$
\left(\begin{array}{cccccccc}
I & 0 & I & 0 & \cdots & I & 0 & I \\
0 & I & 0 & I & \cdots & 0 & I & 0 \\
P & 0 & A_{02} & 0 & \cdots & A_{0, M-2} & 0 & A_{0 M} \\
0 & P & 0 & A_{13} & \cdots & 0 & A_{1, M-1} & 0 \\
\vdots & \vdots & \vdots & \vdots & \vdots & \vdots & \vdots & \vdots \\
0 & 0 & 0 & 0 & \cdots & 0 & A_{M-3, M-1} & 0 \\
0 & 0 & 0 & 0 & \cdots & P & 0 & A_{M-2, M}
\end{array}\right)\left(\begin{array}{c}
X_{0} \\
X_{1} \\
X_{2} \\
X_{3} \\
\vdots \\
X_{M-1} \\
X_{M}
\end{array}\right)=\tilde{F}
$$

where $\tilde{F}=\left(B_{0}, B_{1}, \cdots, B_{M-1}, B_{M}\right)^{\top}$ and $A_{i j}=c_{i} j\left(j^{2}-i^{2}\right) \tilde{I}$ with $\tilde{I}$ being the $(M+$ $1) \times(M+1)$ matrix in Eq. (32) in Appendix.

The solution process and related specific solution form are detailed in Appendix. Although the size of the coefficient matrix in Eq. (18) is $(M+1)^{2} \times(M+1)^{2}$, only the calculation of sub-matrices of order $(M+1) \times(M+1)$ is involved rather than directly inverting the original matrix. Therefore, the proposed Chebyshev spectral method for the 2-D Poisson equation is not only highly accurate but also efficient.

In summary, we evolve the WP system (7) in 4-D phase space as follows

1. Calculate the potential $V_{e}(\boldsymbol{x}, t=0)$ with initial density $n(\boldsymbol{x}, t=0)$ via the $2-\mathrm{D}$ Poisson equation (Eq. (4)) by using Chebyshev spectral method;

2. Using the obtained potential $V_{e}(\boldsymbol{x}, t=0)$ to solve the time-dependent 4-D Wigner equation with operator splitting spectral method to obtain $f(\boldsymbol{x}, \boldsymbol{k}, \Delta t)$ and then to calculate the density $n(\boldsymbol{x}, \Delta t)$ via Eq. (5);

3. Calculate the potential $V_{e}(\boldsymbol{x}, \Delta t)$ with the density $n(\boldsymbol{x}, \Delta t)$, repeat Step (1) and Step (2) until to the final time $t_{f}$. 


\section{Calibration}

In this section, we first would like to verify the convergence rate and efficiency of the proposed solver. The $L^{2}$-error $\varepsilon_{2}(t)$ and $L^{\infty}$-error $\varepsilon_{\infty}(t)$ :

$$
\begin{aligned}
\varepsilon_{2}(t) & =\left(\iint_{\Omega}\left(f^{\text {num }}(\boldsymbol{x}, \boldsymbol{k}, t)-f^{\mathrm{ref}}(\boldsymbol{x}, \boldsymbol{k}, t)\right)^{2} \mathrm{~d} \boldsymbol{x} \mathrm{d} \boldsymbol{k}\right)^{1 / 2}, \\
\varepsilon_{\infty}(t) & =\max _{(\boldsymbol{x}, \boldsymbol{k}) \in \Omega}\left\{\left|f^{\text {num }}(\boldsymbol{x}, \boldsymbol{k}, t)-f^{\mathrm{ref}}(\boldsymbol{x}, \boldsymbol{k}, t)\right|\right\},
\end{aligned}
$$

are employed to study the convergence rate in terms of the number of collocation points and the time step, where $\Omega=\Omega_{k} \times \Omega_{\boldsymbol{x}}$ gives the computational domain in 4 -D phase space, $f^{\text {num }}$ and $f^{\text {ref }}$ denote the numerical solution and reference solution, respectively. To conveniently visualize the 4 -D Wigner function, we plot the reduced 2-D Wigner function [13] in this paper as follows

$$
F(q, k, t):=\iint_{\Omega_{z} \times \Omega_{k_{z}}} \mathrm{~d} z \mathrm{~d} k_{z} f\left(q, z, k, k_{z}, t\right)+\iint_{\Omega_{x} \times \Omega_{k_{x}}} \mathrm{~d} x \mathrm{~d} k_{x} f\left(x, q, k_{x}, k, t\right) .
$$

In addition, the Chebyshev collocation points in $\boldsymbol{x}$-direction for the 4-D Wigner equation and 2-D Poisson keep the same, which may avoid additional interpolations when calculating the electron density $n(\boldsymbol{x}, t)$ in Eq. (4).

\section{- A 2-D Poisson equation}

Consider the Poisson equation in $\Omega_{\boldsymbol{x}}=[-5,5] \times[-5,5]$ with mixed boundary conditions as follows

$$
\begin{aligned}
& \Delta V(x, z)=r(x, z), \\
& \partial_{x} V(-5, z)=g_{1}(z), \quad \partial_{x} V(5, z)=g_{2}(z), \\
& V(x,-5)=g_{3}(x), \quad V(x, 5)=g_{4}(x),
\end{aligned}
$$

the reference solution of which is $V(x, z)=\left(x^{2}+z^{2}\right) \mathbb{e}^{x^{2}+z^{2}}$ and the right terms are

$$
\begin{aligned}
& r(x, z)=\left[4-12\left(x^{2}+z^{2}\right)+4\left(x^{2}+z^{2}\right)^{2}\right] \mathrm{e}^{-\left(x^{2}+z^{2}\right)}, \\
& g_{1}(z)=-g_{2}(z)=10\left(z^{2}+25\right) \mathrm{e}^{-25-z^{2}} \\
& g_{3}(x)=g_{4}(x)=\left(25+x^{2}\right) \mathrm{e}^{-25-x^{2}} .
\end{aligned}
$$

Table 1 gives the calculation time (the second column) and $L^{\infty}$-error (the third column) under the different number of collocation points (the first column). We set $M=8,16,32,64$ and 128 . When $M$ is equal to 64 and 128 , the $L^{\infty}$-error has reached $10^{-14}$, but the calculation time only takes less than 0.1 second. The calculation time in the Table 1 is the serial time with 1 CPU $\left(\right.$ Intel ${ }^{\circledR}$ Core $^{\mathrm{TM}}$ i7-8550U CPU @ 1.80GHz). That is, the proposed Chebyshev spectral method for the 2-D Poisson equation is not only highly accurate but also efficient. The right plot in Table 1 clearly shows the spectral convergence with respect to $M$. 
Table 1: A 2-D Poisson equation: The calculation time (the second column) and $L^{\infty}$ error (the third column) under the different number of collocation points, i.e., $M$ (the first column). The right figure plots the spectral convergence with respect to $M$.

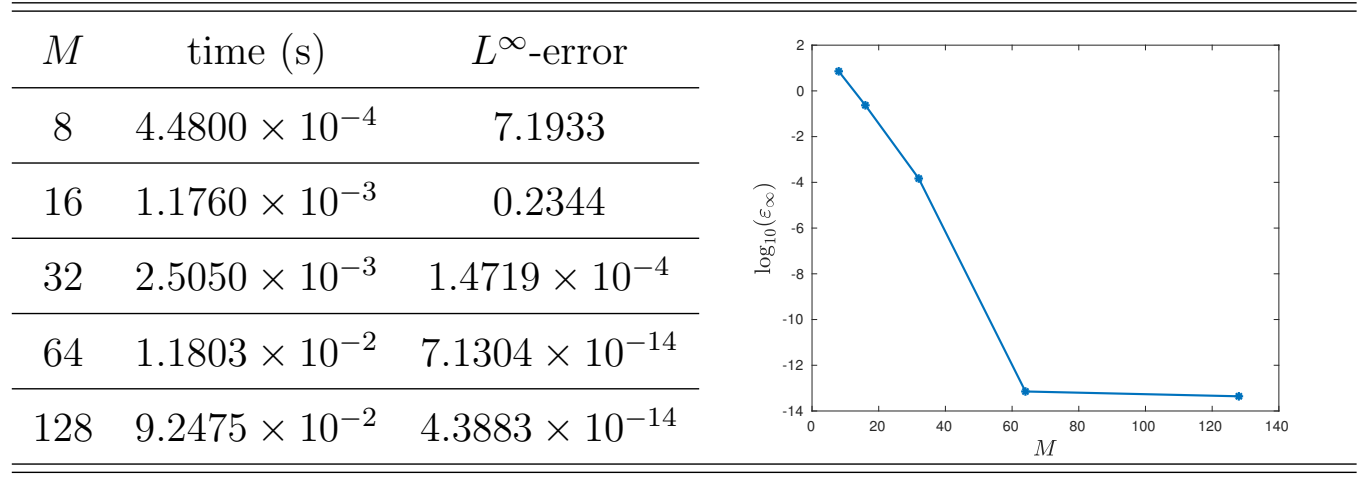

\section{- The WP system in 2-D phase space}

To further validate the overall performance of the operator splitting spectral method for the WP system, we simulate the Gaussian barrier scattering of the Gaussian wave packet $(\mathrm{GWP})[11,13]$ to investigate its convergence rate. We first make tests in 2-D phase space: $\Omega_{k}=\left[-2 \pi \mathrm{nm}^{-1}, 2 \pi \mathrm{nm}^{-1}\right], \Omega_{x}=[-25 \mathrm{~nm}, 25 \mathrm{~nm}]$, and adopt the initial GWP as

$$
f_{0}(x, k)=\frac{1}{\pi} \exp \left[-\frac{\left(x-x_{0}\right)^{2}}{2 a^{2}}-2 a^{2}\left(k-k_{0}\right)^{2}\right],
$$

where $x_{0}$ is the center, $a$ the minimum position spread and $k_{0}$ the initial wavenumber. The Gaussian barrier reads

$$
V_{b}(x)=H \exp \left[-\frac{\left(x-x_{b}\right)^{2}}{2 \omega^{2}}\right]
$$

with $\omega=1 \mathrm{~nm}, x_{b}=0$ and $H=2.3 \mathrm{eV}$. The other parameters are: $x_{0}=-10 \mathrm{~nm}$, $k_{0}=1.4 \mathrm{~nm}^{-1}, a=\sqrt{2} \mathrm{~nm}, \hbar=1 \mathrm{eV} \cdot \mathrm{fs}$, the effective mass $m_{e}=1 \mathrm{eV} \cdot \mathrm{fs}^{2} \cdot \mathrm{nm}^{-2}$ and the final time $t_{f}=20 \mathrm{fs}$. The Poisson equation satisfies the Dirichlet boundary condition with bias potential $V_{0}=0.5 \mathrm{eV}$, the dielectric constant $\epsilon=10 \mathrm{Fm}^{-1}$ and the doping density $N_{d}(x)=0$.

In order to study the convergence rate with respect to $N$ (resp. M), we fix $M=400$ (resp. $N=128$ ) and $\Delta t=0.01 \mathrm{fs}$. As shown in the left and middle plots of Fig. 1, the proposed splitting spectral method shows the spectral convergence with respect to both $N$ and $M$. The right plot of Fig. 1 further displays the fourth-order convergence rate with respect to $\Delta t$ on a fixed mesh $(N, M)=(128,400)$.

Next, we would like to use such Gaussian barrier scattering to study the effect of the space charge on quantum tunneling. The tunneling rate $P_{r}(t)$ [14]

$$
P_{r}(t)=\iint_{\left[0, x_{r}\right] \times \Omega_{k}} f(x, k, t) \mathrm{d} k \mathrm{~d} x
$$

is used to represent the part of GWP passing through the barrier. The mesh is fixed as $(N, M, \Delta t)=(128,256,0.025)$ and other parameters keep unchanged. Fixed $N_{d}=0$ 

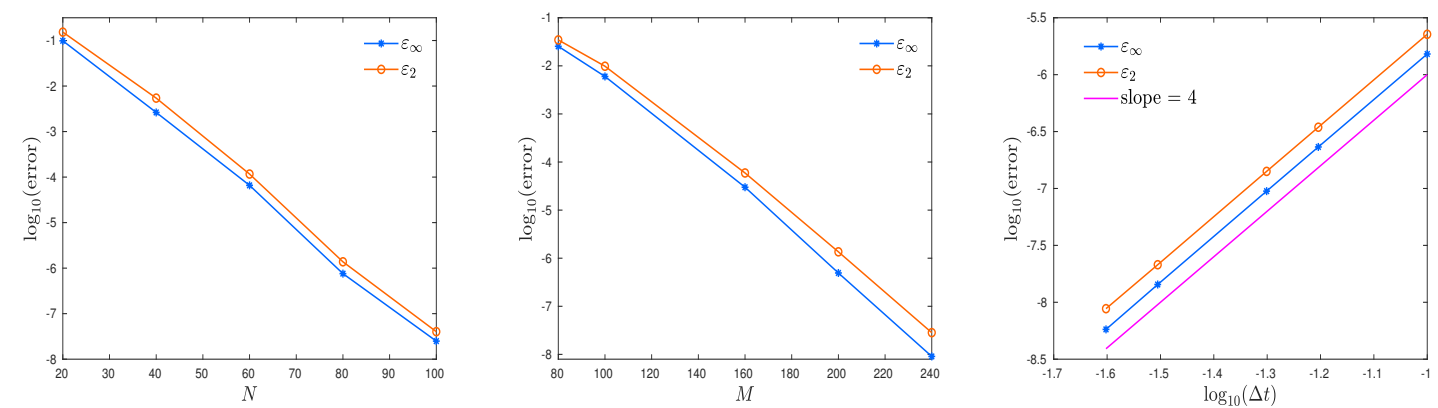

Figure 1: The WP system in 2-D phase space: The convergence rate with respect to $N$ (left) and $M$ (middle) and the time step $\Delta t$ (right). The spectral convergence in both $k$-space and $x$-space, and the fourth-order accuracy against the time step are evident.
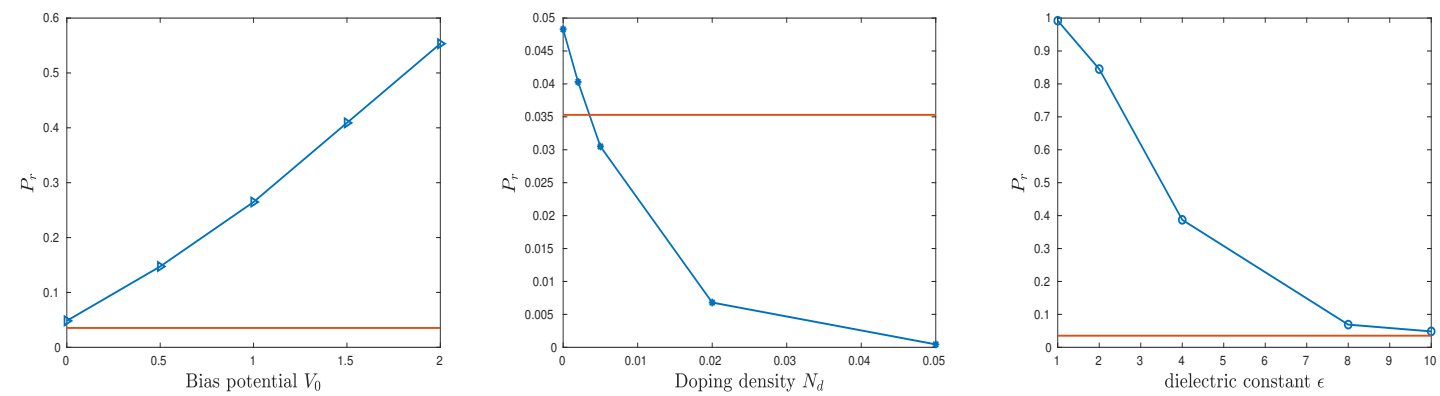

Figure 2: The WP system in 2-D phase space: The red line represents the tunneling rate without coupling the Poisson equation. The blue lines indicate the change of the tunneling rate with the bias potential $V_{0}$ (left), the doping density $N_{d}$ (middle) and the dielectric constant $\epsilon$ (right) when the Poisson equation is coupled.

and $\epsilon=10 \mathrm{Fm}^{-1}$, the tunneling rate is almost proportional to the bias potential $V_{0}$ and higher than the value 0.0353 indicated by the red line, which is the rate for the case without coupling the Poisson equation, as shown in the left plot of Fig. 2. The middle plot of Fig. 2 gives the negative correlation between the tunneling rate and the doping density $N_{d}$ when the bias voltage $V_{0}=0$ and $\epsilon=10 \mathrm{Fm}^{-1}$. The tunneling rate is much smaller than that for the case without coupling the Poisson equation when the doping density gets larger than a certain value (about 0.005). The right plot of Fig. 2 also shows the negative correlation between the tunneling rate and the dielectric constant $\epsilon$, but the rate is always larger than that for the case without coupling the Poisson equation when fixed $V_{0}=0, N_{d}=0$.

We further compare the Wigner functions at instants $t=8,13,18$ fs for only the Wigner equation (left) with those for the WP system (right) in Fig. 3 when setting $V_{0}=0, N_{d}=0, \epsilon=4 \mathrm{Fm}^{-1}$. We are able to clearly see there that it is much easier for GWP to pass through the barrier when the Poisson equation accounting for the space charge effects is coupled.

\section{- The WP system in 4-D phase space}




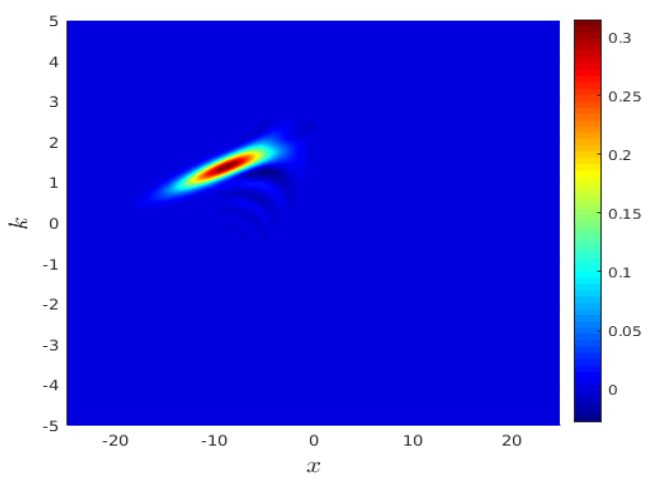

(a) $t=8$, only Wigner equation.

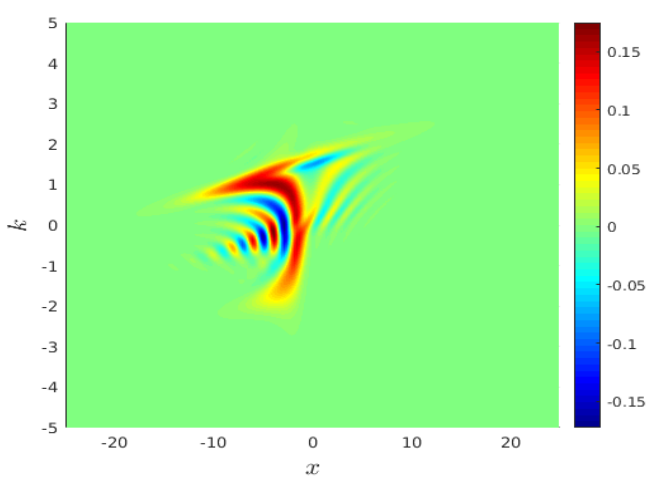

(c) $t=13$, only Wigner equation.

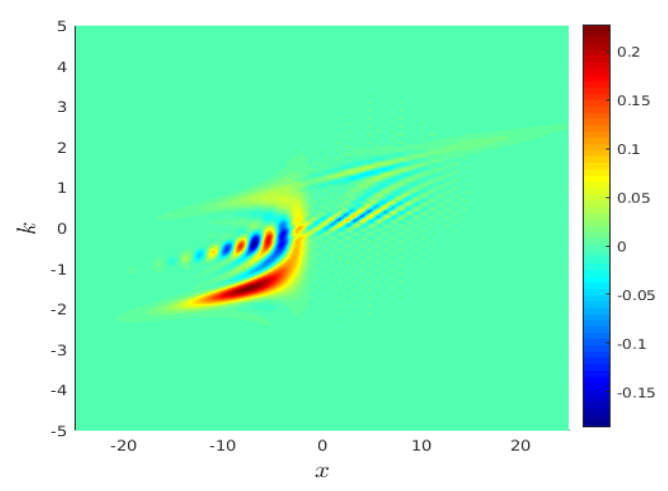

(e) $t=18$, only Wigner equation.

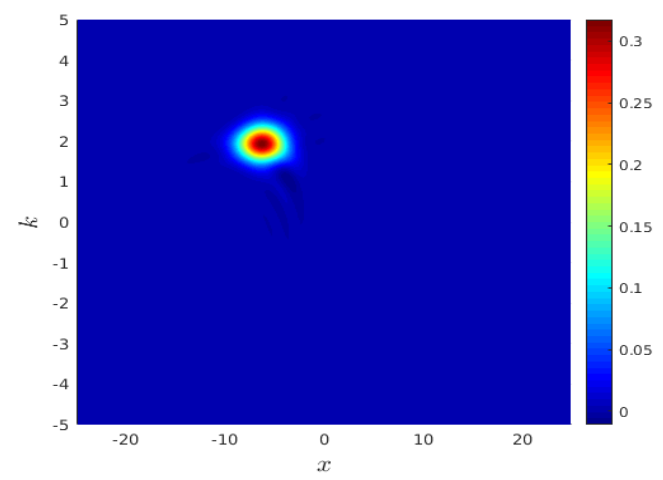

(b) $t=8$, WP system.

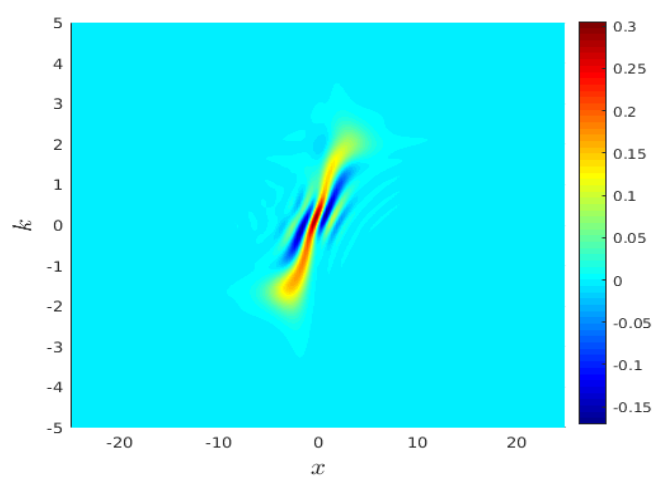

(d) $t=13$, WP system.

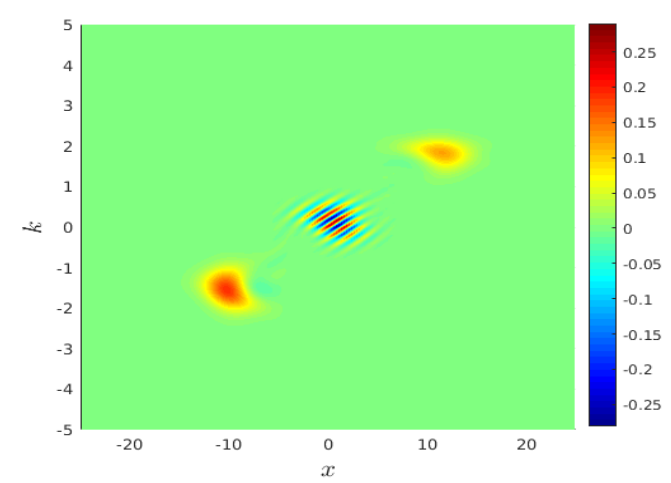

(f) $t=18$, WP system.

Figure 3: The WP system in 2-D phase space: The Wigner functions of only the Wigner equation (left) and the WP system (right). We set $V_{0}=0, N_{d}=0, \epsilon=4 \mathrm{Fm}^{-1}$. It is clearly shown that the space charge effects helps GWP with its tunneling through the barrier.

Now we will calibrate the proposed solver in 4-D phase space still with the Gaussian barrier scattering. We choose the Gaussian barrier as

$$
V_{b}(x, z)=1.3 \exp \left(-x^{2} / 2\right)+1.3 \exp \left(-z^{2} / 2\right),
$$


and the initial GWP as

$$
f_{0}\left(x, z, k_{x}, k_{z}\right)=\frac{1}{\pi^{2}} \exp \left[-\frac{\left(x-x_{0}\right)^{2}}{2 \sigma_{x}^{2}}-2 \sigma_{x}^{2}\left(k_{x}-k_{x}^{0}\right)^{2}-\frac{\left(z-z_{0}\right)}{2 \sigma_{z}^{2}}-2 \sigma_{z}^{2}\left(k_{z}-k_{z}^{0}\right)^{2}\right],
$$

where $x_{0}, z_{0}$ are the center, $k_{x / z}^{0}$ is the initial wavenumber and $\sigma_{x / z}$ is the minimum position spread. We set the parameters to be $\Omega_{\boldsymbol{x}}=[-20 \mathrm{~nm}, 20 \mathrm{~nm}] \times[-20 \mathrm{~nm}, 20 \mathrm{~nm}]$, $\Omega_{k}=\left[-2 \pi \mathrm{nm}^{-1}, 2 \pi \mathrm{nm}^{-1}\right] \times\left[-2 \pi \mathrm{nm}^{-1}, 2 \pi \mathrm{nm}^{-1}\right], \sigma_{x}=\sigma_{z}=1 \mathrm{~nm}, k_{x}^{0}=1.2 \mathrm{~nm}^{-1}$, $k_{z}^{0}=-1.2 \mathrm{~nm}^{-1}$, and $x_{0}=-6 \mathrm{~nm}, z_{0}=6 \mathrm{~nm}$. And we still choose $\hbar=1 \mathrm{eV} \cdot \mathrm{fs}$, $m=1 \mathrm{eV} \cdot \mathrm{fs}^{2} \cdot \mathrm{nm}^{-2}, \epsilon=1 \mathrm{Fm}^{-1}, N_{d}(x)=0$ and $V_{g}=0.5 \mathrm{eV}$.
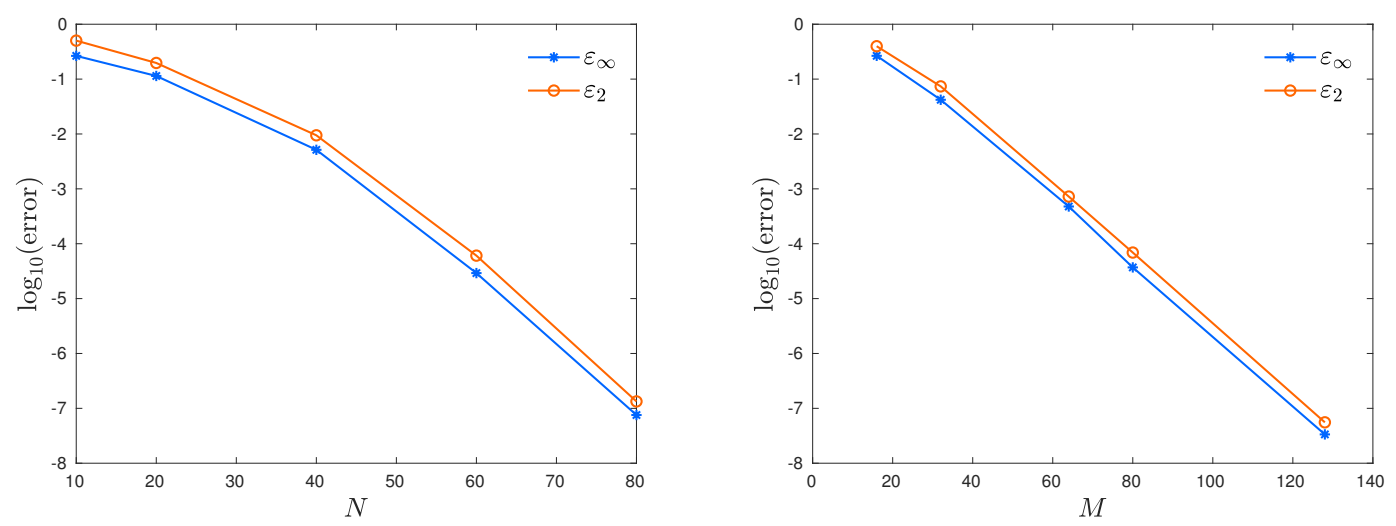

Figure 4: The WP system in 4-D phase space: The convergence rate with respect to $N$ (left) and $M$ (right). The spectral convergence in both $k$-space and $x$-space is clearly shown.

The numerical results are displayed in Fig. 4, where the left (resp. right) plot shows clearly the spectral convergence with respect to $N$ (resp. $M$ ) while fixing $M=200$ (resp. $N=100$ ) and $\Delta t=0.01$ fs. Moreover, we show the reduced Wigner functions of the WP system in 4-D phase space in Fig. 5. It clearly shows that GWP crosses the barrier even when its average kinetic energy $(0.72 \mathrm{eV})$ is lower than the barrier height $(1.3 \mathrm{eV})$ and the Wigner functions obviously have negative values.

\section{Resonant tunneling diode}

As a classical 1-D hetero-structure device with negative differential resistance, RTD exploits resonant tunneling through double barriers as its basic mechanism. Fig. 6 gives a typical type of RTD in which two thin layers (gray) are sandwiched by another three layers (white) to form two energy barriers and one quantum well [8]. In this work, we use constant effective mass $m=0.067 m_{0}$ with $m_{0}$ being the electron mass in vacuum and set the length of the device to $40 \mathrm{~nm}$ which means the computational domain in $x$-space is $\Omega_{x}=[0 \mathrm{~nm}, 40 \mathrm{~nm}]$. The barrier region is set to $3 \mathrm{~nm}$, the length of the quantum well is $4 \mathrm{~nm}$ and the length of the contact is $10 \mathrm{~nm}$. The doping profile in both contacts is depicted as the Fig. 6, where the n-parts are doped with a concentration $2 \times 10^{18} \mathrm{~cm}^{-3}$ and the i-part is doped intrinsically. The 


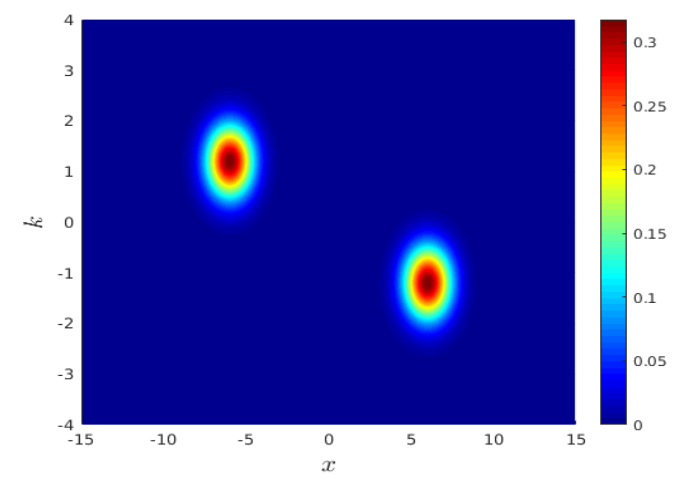

(a) $t=0$.

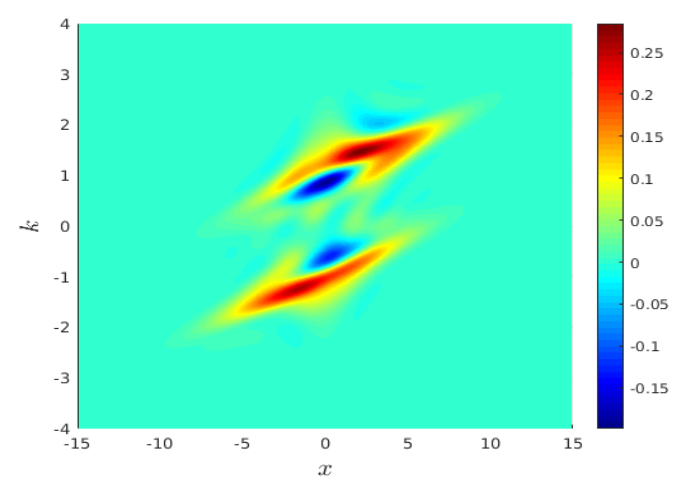

(c) $t=6$.

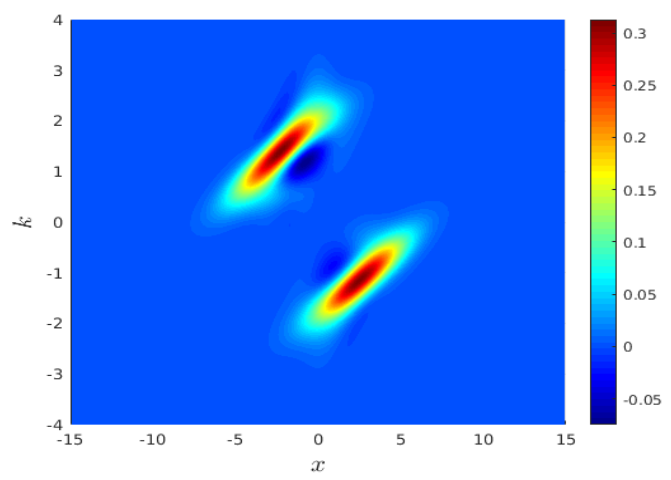

(b) $t=3$.

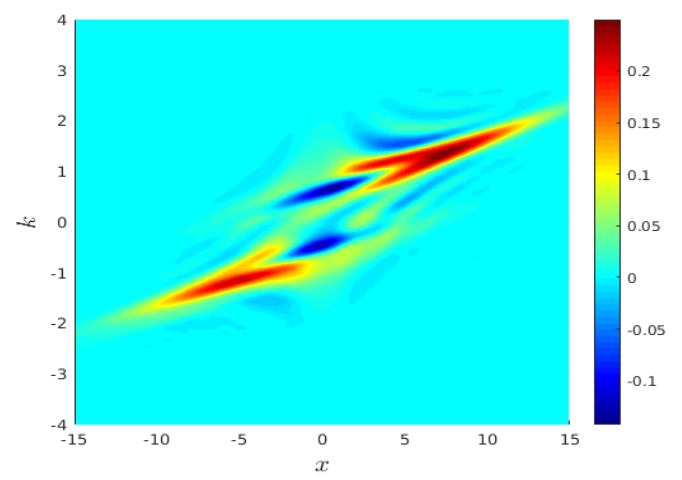

(d) $t=9$.

Figure 5: The WP system in 4-D phase space: The reduced Wigner functions at different instants.

initial and boundary conditions are both taken to be fixed, and given by the shifted equilibrium Fermi-Dirac distribution:

$$
\begin{array}{ll}
f\left(x_{l}, k\right)=\frac{m_{0} k_{B} T}{\pi \hbar} \log \left(1+\exp \left(\frac{\mu_{L}-\hbar^{2}\left(k-k_{0}\right)^{2} / 2 m_{0}}{k_{B} T}\right)\right), & k>0, \\
f\left(x_{r}, k\right)=\frac{m_{0} k_{B} T}{\pi \hbar} \log \left(1+\exp \left(\frac{\mu_{R}-\hbar^{2}\left(k+k_{0}\right)^{2} / 2 m_{0}}{k_{B} T}\right)\right), & k<0,
\end{array}
$$

where $T$ is the temperature, $k_{B}$ is the Boltzmann constant and $\mu_{L}, \mu_{R}$ are the Fermi levels at the left and right contacts, respectively. The parameters are set as: $\Omega_{k}=$ $\left[-\frac{5}{3} \pi \mathrm{nm}^{-1}, \frac{3}{5} \pi \mathrm{nm}^{-1}\right], m_{0}=9.10956 \times 10^{-31} \mathrm{~kg}, \hbar=1.0546 \times 10^{-34} \mathrm{~J} \cdot \mathrm{s}, \varepsilon=$ $15 \mathrm{Fm}^{-1}, k_{B} T=2.5852 \times 10^{-2} \mathrm{eV}$ and $\mu_{L}=\mu_{R}=0.1 \mathrm{eV}$. In order to get rid of possible Gibbs oscillation, a cubic interpolation (smoothing) is used over a unit near the discontinuities in the band potential $V_{b}(x)$ and the doping density $N_{D}(x)$.

We are mostly interested in the formation of steady states of RTD, which correspond formally to the $t \rightarrow+\infty$ limit. Once the steady state is attained, the current of RTD should not appreciably vary with time any longer. To this end, we regard the numerical solution to be the steady state only when the difference in $L^{\infty}$-norm of the electron density given in Eq. (5) between two successive time steps is less than 


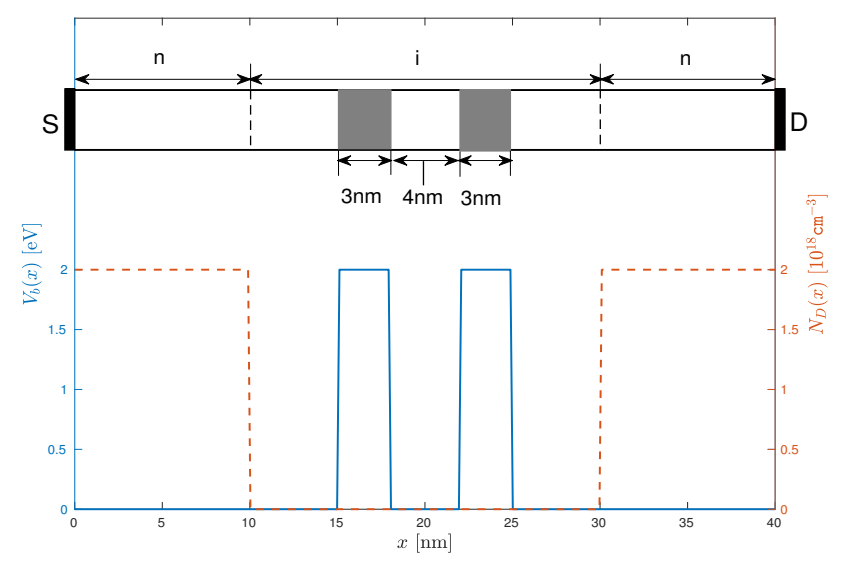

Figure 6: RTD structure: It consists of two n-doped contact with an intrinsic region in between which contains two barriers of $3 \mathrm{~nm}$ wide and height of $1.3 \mathrm{eV}$ with a spacing of 4 $\mathrm{nm}$. The conduction band potential $V_{b}(x)$ (blue solid line) and the doping density $N_{D}(x)$ (red dotted line) are also shown.

$10^{-5}$. Table 2 reports the time to reach the steady states under different number of collocation points $M$ and bias potential $V_{0}(\mathrm{eV})$ when fixing $N=128$ and $\Delta t=0.025$ fs. It can be readily seen there that setting the final time $t_{f}=40 \mathrm{fs}$ is long enough to reach the steady states.

Table 2: RTD: Time (fs) to reach the steady states under different number of collocation points $M$ and bias potential $V_{0}(\mathrm{eV})$ when fixing $N=128$ and $\Delta t=0.025$ fs.

\begin{tabular}{c|ccccc}
\hline \hline & $M=80$ & $M=128$ & $M=160$ & $M=200$ & $M=210$ \\
\hline$V_{0}=0$ & 36.5 & 36.3 & 36.3 & 36.9 & 37.1 \\
\hline$V_{0}=0.2$ & 31.0 & 39.1 & 39.8 & 30.9 & 30.9 \\
\hline$V_{0}=0.5$ & 30.2 & 32.0 & 32.0 & 30.2 & 30.2 \\
\hline$V_{0}=1.0$ & 31.1 & 30.9 & 31.0 & 30.9 & 30.9 \\
\hline \hline
\end{tabular}

In the left plot of Fig. 7 , we show the I-V curves at the final time $t_{f}=40 \mathrm{fs}$ on five different groups of grids: $M=80,128,160,200,210$ after fixing $N=128$ and $\Delta t=0.025 \mathrm{fs}$. First of all, it is evident that the numerical results converge as the resolution increases and the results from small number of grids are unreliable. For example, on the low resolution grid $M=80$, the current density $J$ and the bias potential $V_{b}$ are positively correlated, which is very different from the expected I-V curve. In addition, the current density obtained on the grid $M=128$ has negative values, which is unreasonable. Therefore, high resolution plays a key role in capturing an accurate I-V curve, which constitutes the main reason for us to develop high accurate numerical methods in this paper. Second, the I-V curve in Fig. 7 shows that an incoming distribution of electrons can still generate a current flowing through 

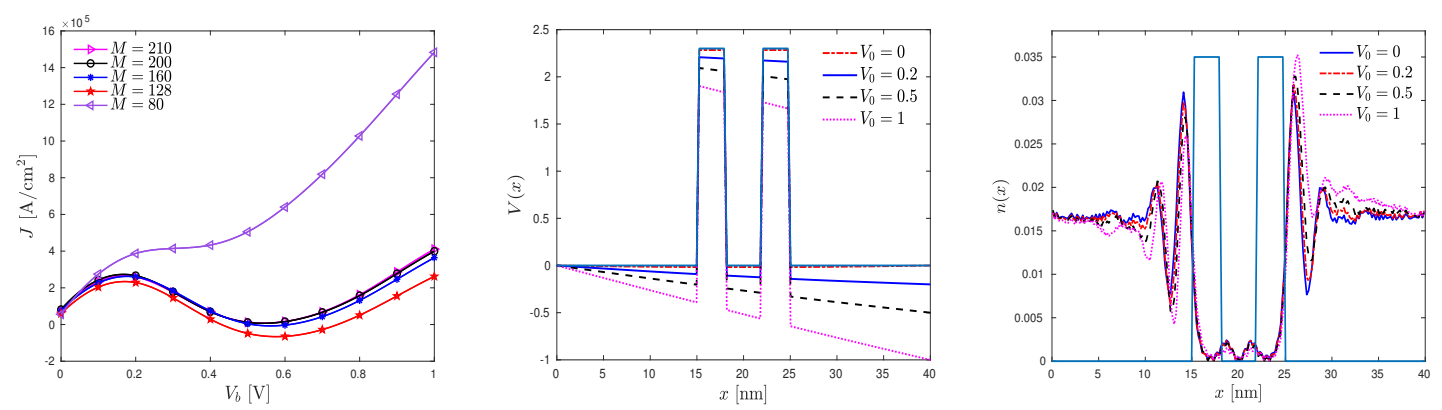

Figure 7: RTD: The I-V curves (left) at $t_{f}=40$ on five different groups of $x$-grids when fixing $N=128$ and $\Delta t=0.025$ fs: $M=80,128,160,200,210$, respectively. The resulting potential $V(x)$ (middle) and the electron density $n(x)$ (right) with different bias potentials: $V_{0}=0,0.2,0.5,1 \mathrm{eV}$ are also shown.

the device even though the number of electrons with a kinetic energy greater than the barrier height is insignificant. Simultaneously, as expected, the current peak is reached when the resonant level in the double well aligns with the energy of the injected electrons. At even higher bias potentials, electrons surmounting the double barriers again increase the current.

In the middle and right plots of Fig. 7 , we further show the converged potential $V(x)$ and electron density $n(x)$ with different bias potentials $V_{0}=0,0.2,0.5,1 \mathrm{eV}$. The height of the barrier decreases with the increase of the bias potential, even if the bias potential is zero. That is, the electric potential $V_{e}$ when taking the space charge effects into account indeed helps the electrons to cross the double barriers.
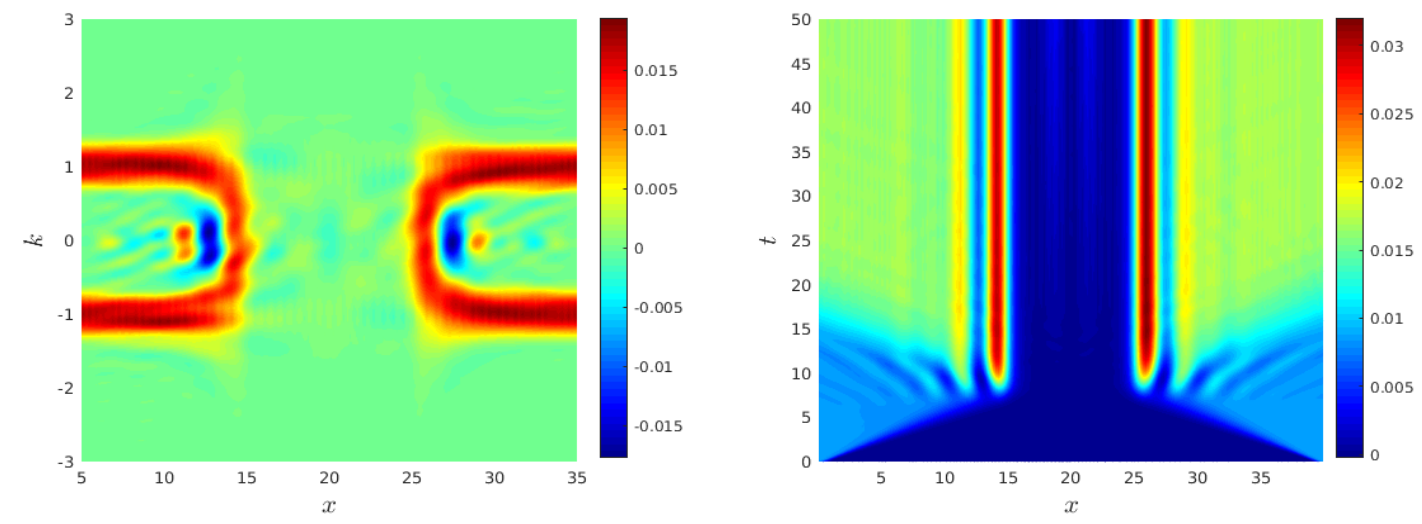

Figure 8: RTD: The Wigner function (left) and the electron density over time (right) near the resonance peak at the bias potential $V_{0}=0.2 \mathrm{eV}$.

Moreover, we show the Wigner function near the resonance peak at the bias potential $V_{0}=0.2 \mathrm{eV}$ in the left plot of Fig. 8. One clearly sees that the Wigner function has negative values, reflecting non-classical behavior. In the right plot of Fig. 8, we also plot the electron density over time. It shows that electrons initially flow from the boundaries into RTD and finally reach a steady state distribution when the simulation time is long enough. 


\section{Double-gate MOSFET}

Fig. 9 cartoons typical structure of dgMOSFET $[15,16]$. The width of the device is assumed to be large, and the potential is invariant along $y$-direction. The silicon layer is sandwiched by two symmetric oxide layers. Source and drain are doped heavily. In this work, the size parameters of the dgMOSFET device are set as follows: the gate length $L_{G}$, equivalent gate oxide thickness EOT and silicon channel thickness $T_{s i}$ are $6 \mathrm{~nm}, 1 \mathrm{~nm}$ and $3 \mathrm{~nm}$, respectively. The highly-doped source and drain access regions are $16 \mathrm{~nm}$ long. The other parameters are: effective mass $m=0.25 m_{0}$, $m_{0}=9.1 \times 10^{-31} \mathrm{~kg}$, dielectric constant $\epsilon=3.9 \epsilon_{0}, \epsilon_{0}=8.85 \times 10^{12} \mathrm{Fm}^{-1}$, doping density $N_{d}=5 \times 10^{19} \mathrm{~cm}^{-3}$ in the highly-doped regions.

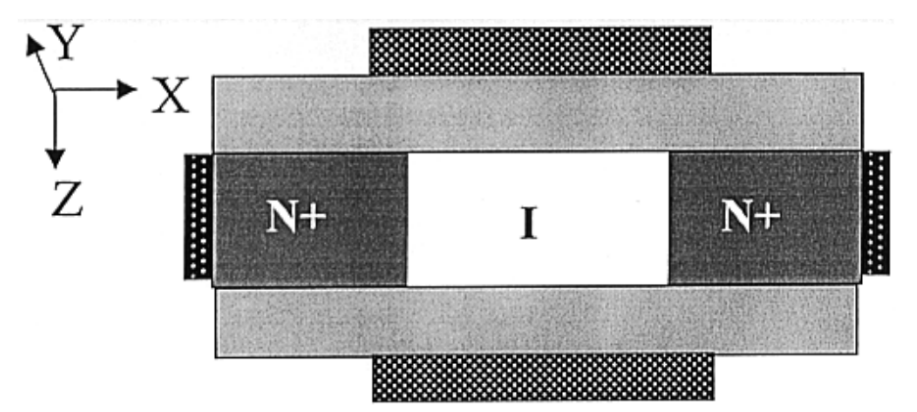

Figure 9: A $16 \mathrm{~nm}$ dgMOSFET structure [15]: The gate length $L_{G}$, equivalent gate oxide thickness EOT and silicon channel thickness $T_{s i}$ are $6 \mathrm{~nm}, 1 \mathrm{~nm}$ and $3 \mathrm{~nm}$, respectively. The source and drain doping is $5 \times 10^{19} \mathrm{~cm}^{-3}$. The transistor is assumed to be wide, i.e., the $y$-direction is treated as infinite long.

The electrons in the real source/drain contacts are in equilibrium characterized by a Fermi level $E_{F 1} / E_{F 2}$. The source level $E_{F 1}$ will be set to be 0 and the Fermi level offset between the source and drain contacts is equal to the applied source/drain bias potential $V_{d s}$, i.e., $E_{F 2}=-V_{d s}$. Consequently, we set the inflow distribution function at $x$-direction to be

$$
\begin{aligned}
& f\left(x_{l}, k_{x}, t\right)=\left[1+\exp \left(\frac{\varepsilon\left(x_{l}\right)-E_{F 1}}{k_{B} T}\right)\right]^{-1}, \quad \text { if } k_{x}>0, \\
& f\left(x_{r}, k_{x}, t\right)=\left[1+\exp \left(\frac{\varepsilon\left(x_{r}\right)-E_{F 2}}{k_{B} T}\right)\right]^{-1}, \quad \text { if } k_{x}<0,
\end{aligned}
$$

where the temperature $T=300 \mathrm{~K}$ and the total energy $\varepsilon\left(x, k_{x}\right)$ of the electron at $\left(x, k_{x}\right)$ is

$$
\varepsilon\left(x, k_{x}\right)=\frac{\hbar^{2}\left(k_{x}-k_{0}\right)^{2}}{2 m}
$$

with $k_{0}=1.4 \mathrm{~nm}^{-1}$ being the initial average wavenumber. And, the initial distribution function at $z$-direction is to be set as a GWP

$$
f\left(z, k_{z}\right)=\exp \left(-k_{z}^{2}\right) \exp \left(-4 z^{2}\right) .
$$


We further set: $\Omega_{\boldsymbol{k}}=\Omega_{k_{x}} \times \Omega_{k_{z}}=\left[-2 \pi \mathrm{nm}^{-1}, 2 \pi \mathrm{nm}^{-1}\right] \times\left[-2 \pi \mathrm{nm}^{-1}, 2 \pi \mathrm{nm}^{-1}\right]$, $\Omega_{\boldsymbol{x}}=\Omega_{x} \times \Omega_{z}=[-8 \mathrm{~nm}, 8 \mathrm{~nm}] \times[-2.5 \mathrm{~nm}, 2.5 \mathrm{~nm}]$, the reduced Planck constant $\hbar=1.0546 \times 10^{-34} \mathrm{~J} \cdot \mathrm{s}$, the time step $\Delta t=0.025 \mathrm{fs}$ and the final time $t_{f}=40 \mathrm{fs}$, which is long enough to reach the steady state.
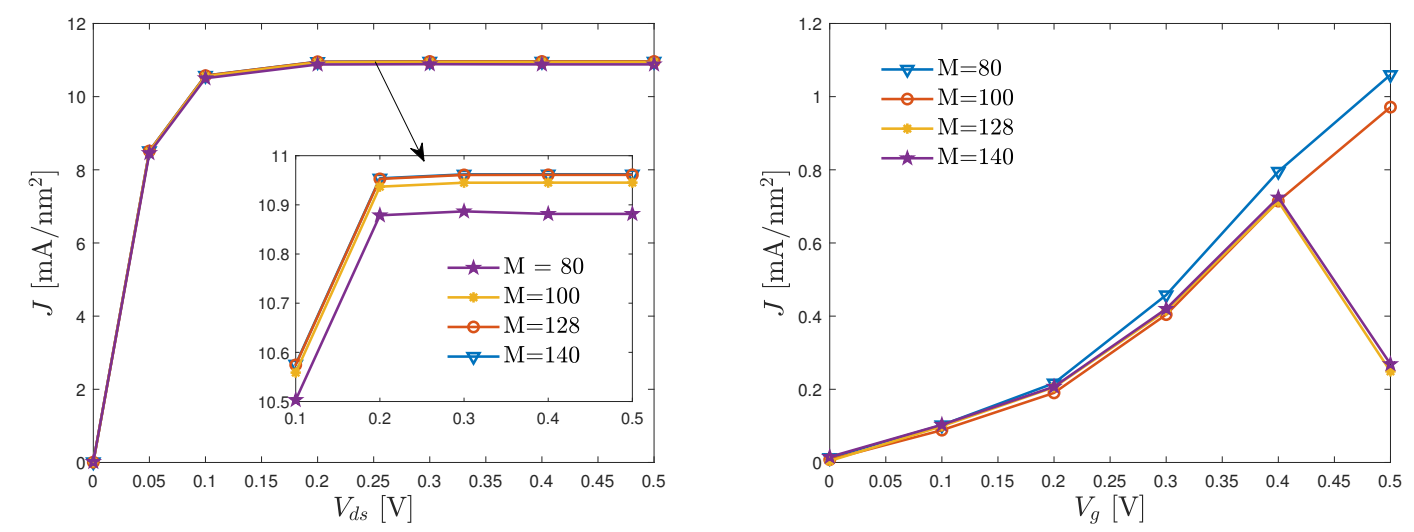

Figure 10: I-V curves in dgMOSFET: Against $V_{d s}$ for $V_{g}=0$ (left) and against $V_{g}$ for $V_{d s}=0$ (right) with $M=80,100,128,140$.

We still investigate the I-V curves but now $V$ contains the gate voltage $V_{g}$ and source/drain bias potential $V_{d s}$. Fig. 10 shows the current density $J$ of steady states against $V_{g}$ and $V_{d s}$, whose $y$-coordinate is the value of $J$ at the center. When $V_{g}=0$ is fixed, the left plot of Fig. 10 gives the I-V curves against $V_{d s}$ which converge as the size of mesh increases (see the inset). Meanwhile, we find that the current density first increases with $V_{d s}$ and then reaches saturation without changing. Similarly, we plot the I-V curves against $V_{g}$ in the right plot of Fig. 10 for fixed $V_{d s}=0$. It is clearly shown that the I-V curves also converge as the mesh becomes denser, and the current density increases first with the increase of $V_{g}$, but decreases after reaching the peak. However, with the lower resolution grids $M=80,100$, the current density continues to increase, as shown in the left plot of Fig. 10. In order to give more details on the steady states, we also plot in Fig. 11 the electron density $n(x, z)$ (first column), reduced Wigner function $f\left(x, k_{x}\right)$ (second column) and electric potential $V_{e}(x, z)$ (third column) under different $V_{d s}$ and $V_{g}$.

\section{Conclusion}

In this paper, we made the first attempt to solve the Winger-Poisson system in 4-D phase space with high accuracy, and succeeded to develop steady states and to obtain numerically converged I-V curves from reliable long-time simulations. We believe that the proposed high-resolution solver may provide more reference solutions to benchmark the stochastic algorithms which have recently attracted a lot of attention due to its simplicity as well as its satisfactory scaling on parallel high-performance machines $[17,18,19,20]$. 

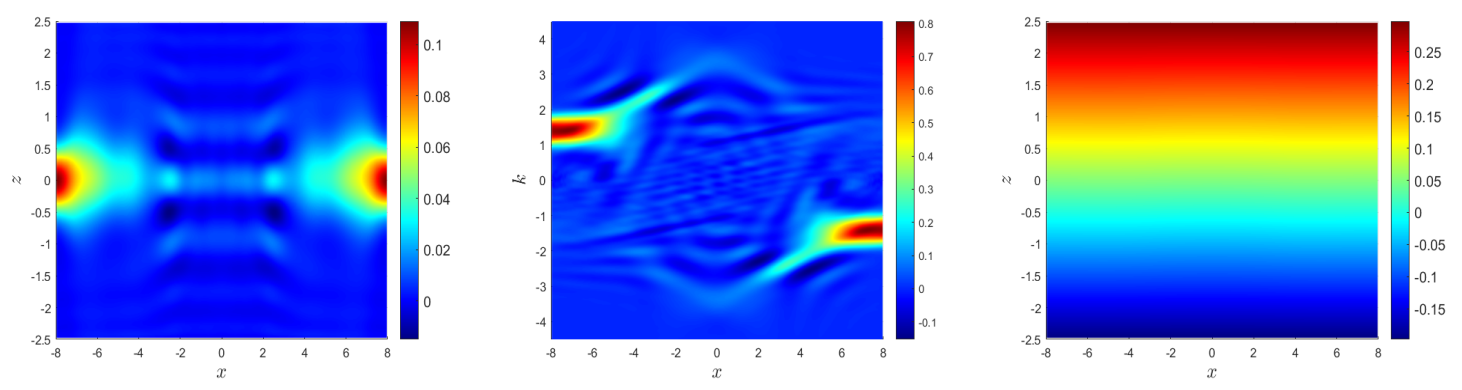

(a) First row: $V_{d s}=0, V_{g}=0.5 \mathrm{eV}$.
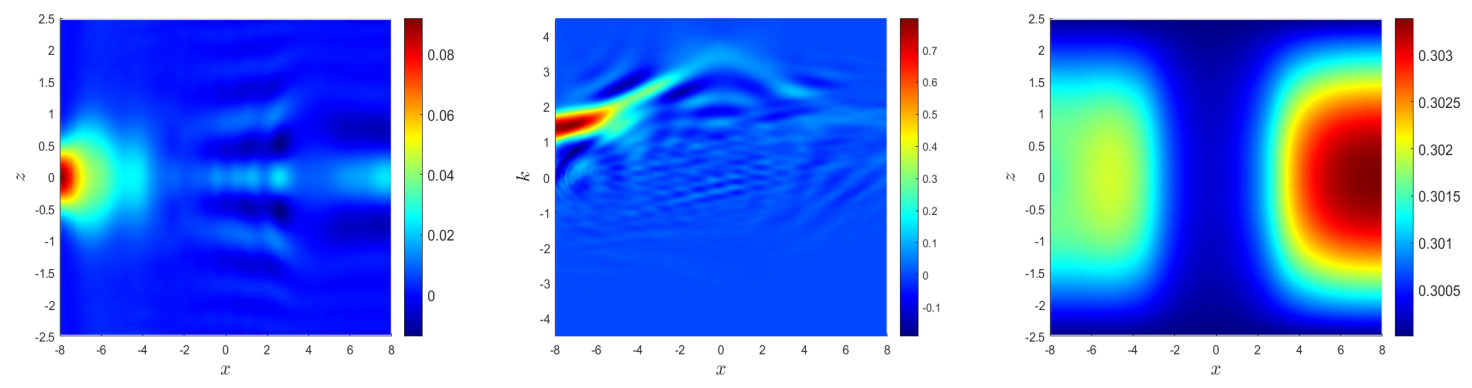

(b) Second row: $V_{d s}=0.5 \mathrm{eV}, V_{g}=0$.

Figure 11: Steady states in dgMOSFET: The electron density $n(x, z)$ (the first column), reduced Wigner function $f\left(x, k_{x}\right)$ (the second column) and electric potential $V_{e}(x, z)$ (the third column). We have set $t_{f}=40 \mathrm{fs}, N=100, M=140$ and $\Delta t=0.025 \mathrm{fs}$.

\section{Acknowledgement}

This work was supported by National Natural Science Foundation of China (No. 11822102). ZC thanks Institute of Applied Physics and Computational Mathematics in Beijing for providing high-performance computing platform. SS acknowledges Beijing Academy of Artificial Intelligence (BAAI) and the computational resource provided by High-performance Computing Platform of Peking University. The authors are grateful to the useful discussions with Haiyan Jiang and Yunfeng Xiong.

\section{Appendix}

We have

$$
P=\left(\begin{array}{cccccccc}
0 & 1 & 0 & 3^{2} & \ldots & 0 & (N-1)^{2} & 0 \\
0 & 0 & 2^{2} & 0 & \ldots & (N-2)^{2} & 0 & N^{2} \\
0 & 0 & \frac{2^{3}}{2} & 0 & \ldots & \frac{(N-2)^{3}}{2} & 0 & \frac{N^{3}}{2} \\
0 & 0 & 0 & 3\left(3^{2}-1\right) & \cdots & 0 & (N-1)\left((N-1)^{2}-1\right) & 0 \\
\vdots & \vdots & \vdots & \vdots & \vdots & \vdots & \vdots & \vdots \\
0 & 0 & 0 & 0 & \cdots & 0 & (N-1)\left((N-1)^{2}-(N-3)^{2}\right) & 0 \\
0 & 0 & 0 & 0 & \cdots & 0 & 0 & N\left(N^{2}-(N-2)^{2}\right)
\end{array}\right)
$$


and

$$
\tilde{I}=\left(\begin{array}{ccccc}
0 & & & & \\
0 & \ddots & & & \\
1 & \ddots & \ddots & & \\
& \ddots & \ddots & \ddots & \\
& & 1 & 0 & 0
\end{array}\right) .
$$

Then, the linear matrix system Eq. (18) equals to the following block upper triangular equation

$$
\left(\begin{array}{cccccccc}
I & 0 & I & 0 & \cdots & I & 0 & I \\
0 & I & 0 & I & \cdots & 0 & I & 0 \\
0 & 0 & \tilde{A}_{02} & 0 & \cdots & \tilde{A}_{0, M-2} & 0 & \tilde{A}_{0 M} \\
0 & 0 & 0 & \tilde{A}_{13} & \cdots & 0 & \tilde{A}_{1, M-1} & 0 \\
\vdots & \vdots & \vdots & \vdots & \vdots & \vdots & \vdots & \vdots \\
0 & 0 & 0 & 0 & \cdots & 0 & \tilde{A}_{M-3, M-1} & 0 \\
0 & 0 & 0 & 0 & \cdots & 0 & 0 & \tilde{A}_{M-2, M}
\end{array}\right)\left(\begin{array}{c}
X_{0} \\
X_{1} \\
X_{2} \\
X_{3} \\
\vdots \\
X_{M-1} \\
X_{M}
\end{array}\right)=\left(\begin{array}{c}
G_{0} \\
G_{1} \\
G_{2} \\
G_{3} \\
\vdots \\
G_{M-1} \\
G_{M}
\end{array}\right),
$$

where

$$
\begin{aligned}
\tilde{A}_{0 i} & =A_{0 j}-P, \quad j=2,4, \ldots, M, \\
\tilde{A}_{1 i} & =A_{1 j}-P, \quad j=3,5, \ldots, M-1, \\
\tilde{A}_{i j} & =A_{i j}-P \tilde{A}_{i-2, i}^{-1} \tilde{A}_{i-2, j}, \quad i=2, \ldots, M-2, \quad j=i+2, \ldots, M, \\
G_{0} & =\tilde{F}_{0}, \\
G_{1} & =\tilde{F}_{1}, \\
G_{2} & =\tilde{F}_{2}-P G_{0}, \\
G_{3} & =\tilde{F}_{3}-P G_{1}, \\
G_{i} & =\tilde{F}_{i}-P \tilde{A}_{i-2, i}^{-1} G_{i-2}, \quad i=4, \ldots, M .
\end{aligned}
$$

Then we get the solution of Eq. (33) as follows

$$
\begin{aligned}
& X_{M}=\left(\tilde{A}_{M-2, M}\right)^{-1} G_{M} \\
& X_{M-1}=\left(\tilde{A}_{M-3, M-1}\right)^{-1} G_{M-1}, \\
& X_{i}=\left(\tilde{A}_{i-2, i}\right)^{-1}\left[G_{i}-\sum_{j=i+2: 2: N} \tilde{A}_{i-2, j} X_{j}\right], i=M-2, \ldots, 2, \\
& X_{1}=G_{1}-\sum_{j=3: 2: M-1} X_{j}, \\
& X_{0}=G_{0}-\sum_{j=2: M} X_{j} .
\end{aligned}
$$




\section{References}

[1] E. Wigner. On the quantum corrections for thermodynamic equilibrium. Phys. Rev., 40:749-759, 1932.

[2] J. Weinbub and D. K. Ferry. Recent advances in Wigner approaches. April, 5:041104, 2018.

[3] M. L. Van de Put, B. Sorée, and W. Magnus. Efficient solution of the WignerLiouville equation using a spectral decomposition of the force field. J. Comput. Phys., 350:314-325, 2017.

[4] A. Gehring and H. Kosina. Wigner function-based simulation of quantum transport in scaled DG-MOSFETs using a Monte Carlo method. J. Comput. Electron., 4:67-70, 2005.

[5] W. R. Frensley. Wigner-function model of a resonant-tunneling semiconductor device. Phys. Rev. B, 36:1570-1580, 1987.

[6] B. A. Biegel. Quantum Electronic Device Simulation. PhD thesis, Stanford University, 1997.

[7] P. Zhao. Wigner-Poisson Simulation of Quantum Devices. PhD thesis, Stevens Institute of Technology, 2000.

[8] H. Jiang, W. Cai, and R. Tsu. Accuracy of the Frensley inflow boundary condition for Wigner equations in simulating resonant tunneling diodes. J. Comput. Phys., 230:2031-2044, 2011.

[9] C. Ringhofer. A spectral collocation technique for the solution of the WignerPoisson problem. SIAM J. Numer. Anal., 29:679-700, 1992.

[10] A. Arnold and C. Ringhofer. A operator splitting method for the Wigner-Poisson problem. SIAM J. Numer. Anal., 33:1622-1643, 1996.

[11] Z. Chen, S. Shao, and W. Cai. A high order efficient numerical method for 4-D Wigner equation of quantum double-slit interferences. J. Comput. Phys., 396:54-71, 2019.

[12] S. Shao, T. Lu, and W. Cai. Adaptive conservative cell average spectral element methods for transient Wigner equation in quantum transport. Commun. Comput. Phys., 9:711-739, 2011.

[13] Y. Xiong, Z. Chen, and S. Shao. An advective-spectral-mixed method for timedependent many-body Wigner simulations. SIAM J. Sci. Comput., 38:B491B520, 2016.

[14] Z. Chen, Y. Xiong, and S. Shao. Numerical methods for the Wigner equation with unbounded potential. J. Sci. Comput., 79:345-368, 2019. 
[15] Z. Ren, R. Venugopal, S. Goasguen, S. Datta, and M. S. Lundstrom. nanoMOS 2.5: A two-dimensional simulator for quantum transport in double-gate MOSFETs. IEEE Trans. Electron Devices, 50:1914-1925, 2003.

[16] D. Querlioz, J. Saint-Martin, V. Do, A. Bournel, and P. Dollfus. A study of quantum transport in end-of-roadmap DG-MOSFETs using a fully self-consistent Wigner Monte Carlo approach. IEEE Trans. Nanotechnol., 5:737-744, 2006.

[17] O. Muscato and W. Wagner. A class of stochastic algorithms for the Wigner equation. SIAM J. Sci. Comput., 38:A1483-A1507, 2016.

[18] Y. Xiong and S. Shao. The Wigner branching random walk: Efficient implementation and performance evaluation. Commun. Comput. Phys., 25:871-910, 2019.

[19] M. Nedjalkov, I. Dimov, and S. Selberherr. Stochastic approaches to electron transport in micro- and nanostructures. Birkhäuser, Switzerland, 2021.

[20] M. Benam M. Ballicchia, J. Weinbub, S. Selberherr, and M. Nedjalkov. A Wigner potential decomposition in the signed-partical Monte Carlo approach. J. Comput. Electron., 20:775-784, 2021. 